J. Korean Math. Soc. 49 (2012), No. 3, pp. 571-583

http://dx.doi.org/10.4134/JKMS.2012.49.3.571

\title{
SHIFT GENERATED DUAL FRAMES FOR LOCALLY COMPACT ABELIAN GROUPS
}

\author{
Ahmad Ahmadi and Ataollah Askari-Hemmat
}

\begin{abstract}
Let $G$ be a metrizable, $\sigma$-compact locally compact abelian group with a compact open subgroup. In this paper we define the Gramian and the dual Gramian operators for shift invariant subspaces of $L^{2}(G)$ and we use them to characterize shift generated dual frames for shift invariant spaces, which forms a frame for a subspace of $L^{2}(G)$. We present necessary and sufficient conditions for which standard dual is a unique SG-dual frame of type I and type II.
\end{abstract}

\section{Introduction and preliminaries}

Dual frames are useful tools for construction of series expansion in a Hilbert space. The coefficients in these series may not be unique. This property has many applications such as in noise reduction or for the reconstruction from lossy data $[7,16]$. The theory of frames goes back to Duffin and Schaeffer [8]. In this paper we want to characterize shift generated dual frames for shift invariant spaces which forms a frame for closure of its span. We borrow ideas of [12] and state them in terms of locally compact abelian (LCA) groups. First, we will recall some definitions about frames and Riesz basis (for more details see $[6])$.

Definition 1.1. Let $\mathcal{H}$ be a Hilbert space. A subset $F=\left\{f_{n}\right\}_{n \in \mathbb{N}} \subseteq \mathcal{H}$ is called a frame if there exist two numbers $0<A \leq B<\infty$ such that

$$
A\|f\|^{2} \leq \sum_{n \in \mathbb{N}}\left|\left\langle f, f_{n}\right\rangle\right|^{2} \leq B\|f\|^{2} \text { for all } f \in \mathcal{H} .
$$

If $A=B$, then $F$ is called a tight frame. The collection $F$ is called a frame sequence, if $F$ is a frame for $\operatorname{span}(F)=\mathcal{M}$. The set $F$ is called Bessel with Bessel bound $B$, if the right-hand inequality in (1.1) holds.

Received July 8, 2010; Revised November 19, 2011.

2010 Mathematics Subject Classification. Primary 42C40; Secondary 11S85.

Key words and phrases. frames, Gramian operator, locally compact abelian group, shift invariant space, SG-dual frame. 
A subset $F=\left\{f_{n}\right\}_{n \in \mathbb{N}} \subseteq \mathcal{H}$ is called a Riesz family for $\overline{\operatorname{span}}(F)=\mathcal{M}$ if there exist two numbers $0<A \leq B<\infty$ so that

$$
A \sum_{n \in \mathbb{N}}\left|h_{n}\right|^{2} \leq\left\|\sum_{n \in \mathbb{N}} h_{n} f_{n}\right\|^{2} \leq B \sum_{n \in \mathbb{N}}\left|h_{n}\right|^{2}
$$

for all finitely supported $\left\{h_{n}\right\}_{n \in \mathbb{N}} \subseteq \mathbb{C}$. If $\overline{\operatorname{span}}(F)=\mathcal{H}$, we say $F$ is Riesz basis. For Bessel collection $F=\left\{f_{n}\right\}_{n \in \mathbb{N}}$, we recall the definitions of analysis operator $T_{F}: \mathcal{M} \rightarrow l^{2}(\mathbb{N})$ by $T_{F}(f)=\left\{\left\langle f, f_{n}\right\rangle\right\}_{n \in \mathbb{N}}, f \in \mathcal{M}$. The synthesis operator for $F$ is defined by $T_{F}^{*}: l^{2}(\mathbb{N}) \rightarrow \mathcal{M}$ by $T_{F}^{*}\left(\left\{c_{n}\right\}_{n \mathbb{N}}\right)=\sum_{n \in \mathbb{N}} c_{n} f_{n}$, $\left\{c_{n}\right\}_{n \in \mathbb{N}} \in l^{2}(\mathbb{N})$. The operator $S=T_{F}^{*} T_{F}: \mathcal{M} \rightarrow \mathcal{M}$ is called the frame operator. If $F$ is a frame, then $S$ is a bounded and invertible operator from $\mathcal{M}$ onto $\mathcal{M}$. The collection $\left\{S^{-1} f_{n}\right\}_{n \in \mathbb{N}}$ is called the standard dual frame of frame $F$.

Now, we are ready to recall three types of dual frame which have been defined in $[12]$.

Definition 1.2. Let $F=\left\{f_{n}\right\}_{n \in \mathbb{N}}$ be a frame for the closed subspace $\mathcal{M}$ of the Hilbert space $\mathcal{H}$ with inner product denoted by $\langle\cdot, \cdot\rangle$.

(1) A dual frame for the frame $F$ is a Bessel collection $K=\left\{k_{n}\right\}_{n \in \mathbb{N}} \subseteq \mathcal{H}$ satisfying $\sum_{n \in \mathbb{N}}\left\langle f, k_{n}\right\rangle f_{n}=f, f \in \mathcal{M}$.

(2) A dual frame of type I for the frame $F$ is a dual $\left\{k_{n}\right\}_{n \in \mathbb{N}}$ such that $k_{n} \in \mathcal{M}$ for each $n \in \mathbb{N}$.

(3) A dual frame of type II for the frame $F$ is a dual $K=\left\{k_{n}\right\}_{n \in \mathbb{N}}$ with the property Range $\left(T_{K}\right) \subset$ Range $\left(T_{F}\right)$, where $T_{F}$ and $T_{K}$ denotes the analysis operators associated with $F$ and $K$, respectively.

Throughout this paper we assume that $G$ is an LCA group with a compact open subgroup $H$ and Haar measure $\mu$ such that $\mu(H)=1$, and the dual group $\hat{G}$ with Haar measure $\nu$ such that $\nu\left(H^{\perp}\right)=1$, where $H^{\perp}=\{\gamma \in \hat{G}: \quad(x, \gamma)=$ 1 for all $x \in H\} \subseteq \hat{G}$ and $(x, \gamma)$ denotes the action of the duality between $G$ and $\hat{G}$. By Theorem 5.21 of [13], $G / H$ and $\hat{G} / H^{\perp}$ are discrete, thus $\mu_{G / H}$ and $\nu_{\hat{G} / H^{\perp}}$ are counting measures. For more details about LCA groups we refer to [9], [13] and [17]. $G$ is metrizable if and only if $\hat{G}$ is $\sigma$-compact [17]. Also if $G$ is $\sigma$-compact, then $G / H$ is countable. In this paper we consider metrizable, $\sigma$-compact LCA group $G$. A good example for this situation is $Q_{p}$, that is the completion of $\mathbb{Q}$ with respect to a certain natural metric topology. One of the most important application of these groups is in quantum physics [10]. The Fourier transform $\wedge: L^{1}(G) \rightarrow \mathcal{C}_{0}(\hat{G})$, is defined by

$$
\wedge(f)(\gamma)=\hat{f}(\gamma)=\int_{G} f(x) \overline{(x, \gamma)} d \mu(x) .
$$

The identity $\int_{G} f(x) d \mu(x)=\int_{G / H} \int_{H} f(x+y) d \mu(y) d \mu_{G / H}(x H)$ for $f \in L^{1}(G)$ is called Weil's Formula, which in fact, determines the relation between the 
Haar measures of $\mathrm{G}$ and $G / H$. The multiplication operator on $L^{2}(\hat{G})$ and the translation and dilation operators on $L^{2}(G)$ have been defined in [3] and [4].

Definition 1.3. Let $G$ be an LCA group with compact open subgroup $H$, let $\mathcal{D} \subseteq \hat{G}$ be the set of coset representatives in $\hat{G}$ for the quotient $\hat{G} / H^{\perp}$.

a) The maps $\theta=\theta_{D}: \hat{G} \rightarrow \mathcal{D}$ and $\eta=\eta_{D}: \hat{G} \rightarrow H^{\perp}$ are defined by: $\theta(\gamma)=$ the unique $\sigma_{\gamma} \in \mathcal{D}$ and $\eta_{\mathcal{D}}(\gamma)=\gamma-\theta(\gamma)$.

b) For any fixed $[s] \in G / H$ we define the unimodular weight function

$$
\omega_{[s]}(\gamma)=\overline{\left(s, \eta_{\mathcal{D}}(\gamma)\right)}
$$

c) For any fixed $[s] \in G / H$ we define the multiplier $m_{[s]}$ on $L^{2}(\hat{G})$ as multiplication by $\omega_{[s]}$, that is, for any $F \in L^{2}(\hat{G}), m_{[s]} F(\gamma)=F(\gamma) \omega_{[s]}(\gamma)$.

d) For any fixed $[s] \in G / H$ and for any $f \in L^{2}(G)$, we define $\tau_{[s]} f$ to be the inverse Fourier transform of $m_{[s]} \hat{f}$, that is, $\tau_{[s]} f=f * \check{\omega}_{[s]}$, where $\check{\omega}_{[s]}$ is the inverse Fourier transform of $\omega_{[s]}$.

The theory of shift invariant spaces in $L^{2}(\mathbb{R})$ was presented by Helson [11] and characterized in $L^{2}\left(\mathbb{R}^{n}\right)$ by Bownik in [5]. In [14], $\mathbb{R}^{n}$ is replaced by $G$, where $G$ is an LCA group with a uniform lattice (a cocompact discrete subgroup). The authors have been defined shift invariant spaces for LCA groups with a compact open subgroup which we recall that in the following definition.

Definition 1.4. A closed subspace $V \subseteq L^{2}(G)$ is called shift invariant space with respect to $G / H$, if $f \in V$ implies $\tau_{[s]} f \in V$ for all $[s] \in G / H$, where $\tau_{[s]}$ is the translation operator defined in Definition 1.3. For countable subset $\Phi \subset L^{2}(G)$ we define $V_{\Phi}=\overline{\operatorname{span}}\left\{\tau_{[s]} \phi:[s] \in G / H \quad \phi \in \Phi\right\}$.

If the shift invariant system $X$ is a frame for $\mathcal{M}$ but is not a Riesz family, then there exists a dual frame except standard dual for $X$, which is shift invariant. We define three types of shift generated dual for shift invariant space $X$.

Definition 1.5. Let $X=\left\{\tau_{[s]} \phi ; \quad[s] \in G / H, \phi \in \Phi\right\}$ be a frame for closed subspace $V_{\Phi} \subset L^{2}(G)$. Let $R: \Phi \rightarrow L^{2}(G)$ be a mapping and $Y=\left\{\tau_{[s]} R \phi ; \quad[s] \in\right.$ $G / H, \phi \in \Phi\}$.

(i) We say that $Y$ is shift generated $(S G)$-dual frame for $X$ if it is a dual frame for $X$ as in Definition 1.2(1).

(ii) We say that $Y$ is shift generated $(S G)$-dual frame of type $I$ (resp. type $I I)$ for $X$, if it is a dual frame for $X$ as in Definition 1.2(2) (resp. (3)).

We consider $l_{\perp}^{2}:=l^{2}\left(\hat{G} / H^{\perp}\right)$ and define the Hilbert space

$$
L_{*}^{2}:=L^{2}\left(H^{\perp}, l_{\perp}^{2}\right)=\left\{\Phi: H^{\perp} \rightarrow l_{\perp}^{2} ; \int_{H^{\perp}}\|\Phi(\gamma)\|_{l_{\perp}^{2}}^{2} d \nu(\gamma)<\infty\right\},
$$

with inner product $\langle f, g\rangle=\int_{H^{\perp}}\langle f(\xi), g(\xi)\rangle_{l_{\perp}^{2}} d \nu(\xi)$, and norm

$$
\|f\|_{L_{*}^{2}}=\int_{H^{\perp}}\|f(\xi)\|_{l_{\perp}^{2}}^{2} d \nu(\xi) .
$$


Also, the mapping $\digamma: L^{2}(G) \rightarrow L_{*}^{2}$ defined by $(\digamma g)(\gamma)=\{\hat{g}(\gamma+\eta)\}_{[\eta] \in \hat{G} / H^{\perp}}$ is an isometric isomorphism between $L^{2}(G)$ and $L_{*}^{2}$ (for more details see $[2,15]$ ).

Definition 1.6. A range function is a mapping

$$
J: H^{\perp} \rightarrow\left\{\text { closed subspaces of } l_{\perp}^{2}\right\} .
$$

The mapping $J$ is called measurable if the orthogonal projections

$$
P(\xi): l_{\perp}^{2} \rightarrow J(\xi) \text { for a.e. } \xi \in H^{\perp}
$$

are weakly measurable, i.e., $\xi \rightarrow\langle P(\xi) a, b\rangle$ are measurable for all $a, b \in l_{\perp}^{2}$.

Proof of Theorems 1.7 and 1.8 are similar to proof of Theorems 3.1 of [15] and 2.3 of [5] respectively which have been proved by authors in [2].

Theorem 1.7. Let $G$ be an LCA group with compact open subgroup $H$. $A$ closed subspace $V \subseteq L^{2}(G)$ is shift invariant, with respect to the lattice induced by $G / H$, if and only if

$$
V=\left\{f \in L^{2}(G) \quad \digamma f(\xi) \in J(\xi) \text { for a.e. } \xi \in H^{\perp}\right\},
$$

where $J$ is a measurable range function. Moreover, if $V_{\Phi}$ is generated by some countable set $\Phi \subset L^{2}(G)$, then $J(\xi)=\overline{\operatorname{span}}\{\digamma \phi(\xi) ; \phi \in \Phi\}$.

Theorem 1.8. Suppose $G$ is a metrizable LCA group with a compact open subgroup $H$ and $\Phi \subset L^{2}(G)$ is a countable set. Then $\left\{\tau_{[s]} \phi: \phi \in \Phi,[s] \in G / H\right\}$ is a frame (Riesz family) for $V_{\Phi}$ with bounds $A$ and $B$ if and only if $\{\digamma \phi(\xi)$ : $\phi \in \Phi\}$ is a frame (Riesz family) for $J(\xi)$ a.e. $\xi \in H^{\perp}$.

This paper is organized in three sections, this section consists of definitions and theorems that we need. In Section 2, we define the Gramian and the dual Gramian operators for shift invariant subspaces of $L^{2}(G)$ and using them to characterize frame and Riesz basis. Finally, in Section 3, we characterize SGdual frames and we state necessary and sufficient conditions for standard dual to be unique dual of type I or type II.

\section{The Gramian operator}

In this section we define the Gramian operator and its dual for shift invariant subspaces of $L^{2}(G)$, where $G$ is an LCA group with a compact open subgroup. For a countable subset $\Phi \subset L^{2}(G)$ we assume that $X=\left\{\tau_{[s]} \phi ; \quad[s] \in G / H, \phi \in\right.$ $\Phi\}$ is a shift invariant subspace of $L^{2}(G)$. Fix $\xi \in H^{\perp}$, the pre-Gramian operator $K(\xi): l^{2}(\Phi) \rightarrow l_{\perp}^{2}$, is defined by $K(\xi)\left(\left\{c_{\phi}\right\}_{\phi \in \Phi}\right)=\sum_{\phi \in \Phi} c_{\phi} \digamma \phi(\xi)$.

If $X$ has Bessel property with Bessel bound $B$, then $K(\xi)$ is bounded and $\|K(\xi)\|^{2} \leq B$, and its adjoint, $K^{*}(\xi): l_{\perp}^{2} \rightarrow l^{2}(\Phi)$, is given by $K^{*}(\xi)(b)=$ $\{\langle b, \digamma \phi(\xi)\rangle\}_{\phi \in \Phi \text {. }}$

Let $\xi \in H^{\perp}$, we define the Gramian operator, $\mathbb{G}(\xi): l^{2}(\Phi) \rightarrow l^{2}(\Phi)$ by $\mathbb{G}(\xi)=K^{*}(\xi) K(\xi)$ and the dual Gramian operator, $\widetilde{\mathbb{G}}(\xi): l_{\perp}^{2} \rightarrow l_{\perp}^{2}$ by $\widetilde{\mathbb{G}}(\xi)=$ $K(\xi) K^{*}(\xi)$. 
Thus the entries of the Gramian operator and the dual Gramian operator are define by

$$
\mathbb{G}_{\phi_{1}, \phi_{2}}(\xi)=\sum_{[\eta] \in \hat{G} / H^{\perp}} \hat{\phi}_{1}(\xi+\eta) \overline{\hat{\phi}_{2}(\xi+\eta)}=\left\{\left\langle\digamma \phi_{1}(\xi), \digamma \phi_{2}(\xi)\right\rangle\right\}_{\phi_{1}, \phi_{2} \in \Phi},
$$

and $\quad \widetilde{\mathbb{G}}_{\left[\eta_{1}\right],\left[\eta_{2}\right]}(\xi)=\sum_{\phi \in \Phi} \hat{\phi}\left(\xi+\eta_{1}\right) \overline{\hat{\phi}\left(\xi+\eta_{2}\right)}$.

Remark 2.1. (1) By Weil's Formula and Cauchy inequality we have

$$
\begin{aligned}
& \int_{H^{\perp}}\left|\sum_{[\eta] \in \hat{G} / H^{\perp}} \hat{\phi}_{i}(\xi+\eta) \overline{\hat{\phi}_{j}(\xi+\eta)}\right| d \nu(\xi) \\
\leq & \left(\int_{H^{\perp}} \sum_{[\eta] \in \hat{G} / H^{\perp}}\left|\hat{\phi}_{i}(\xi+\eta)\right|^{2} d \nu(\xi)\right)^{\frac{1}{2}}\left(\int_{H^{\perp}} \sum_{[\eta] \in \hat{G} / H^{\perp}}\left|\hat{\phi}_{j}(\xi+\eta)\right|^{2} d \nu(\xi)\right)^{\frac{1}{2}} \\
= & \left\|\phi_{i}\right\|\left\|\phi_{j}\right\|<\infty .
\end{aligned}
$$

Thus the entries $\mathbb{G}(\xi)$ are well defined.

(2) If $\sum_{\phi \in \Phi}|\hat{\phi}(\xi+\eta)|^{2}<\infty$, then the entries of $\widetilde{\mathbb{G}}(\xi)$ are well defined.

(3) We have

$$
\|\mathbb{G}(\xi)\|=\left\|K^{*}(\xi)\right\|^{2} \text { for a.e. } \xi \in H^{\perp} .
$$

(4) For a.e. $\xi \in H^{\perp}, \mathbb{G}(\xi)=0$ if and only if $J(\xi)=0$. Indeed, $\mathbb{G}(\xi)=0$ if and only if $\mathbb{G}_{\phi_{1}, \phi_{2}}(\xi)=0$ for all $\phi_{1}, \phi_{2} \in \Phi$. Now if $\phi_{1}=\phi_{2}=\phi$ we have $\sum_{[\eta] \in \hat{G} / H^{\perp}}|\hat{\phi}(\xi+\eta)|^{2}=0$. Thus $\phi=0$ for all $\phi \in \Phi$, which shows that $J(\xi)=0$ for a.e. $\xi \in H^{\perp}$. Conversely if $J(\xi)=0$, then $\digamma \phi(\xi)=0$ for all $\phi \in \Phi$ and for a.e. $\xi=H^{\perp}$. Thus $\mathbb{G}(\xi)=0$. By similar way, for a.e. $\xi \in H^{\perp}, \widetilde{\mathbb{G}}(\xi)=0$ if and only if $J(\xi)=0$.

In the following theorem we use the Gramian operator and state a necessary and sufficient condition for the shifts of a countable subset of $L^{2}(G)$ has Bessel property.

Theorem 2.2. The system $X=\left\{\tau_{[s]} \phi ; \quad[s] \in G / H, \phi \in \Phi\right\}$ to have the Bessel property with Bessel constant $B$ if and only if $\operatorname{essup}_{\xi \in H^{\perp}}\|\mathbb{G}(\xi)\| \leq B$.

Proof. The set $\left\{\digamma \tau_{[s]} \phi(\xi): \phi \in \Phi\right\}$ is a Bessel family for $J(\xi)$ a.e. $\xi \in H^{\perp}$ if and only if $\left\|K^{*}(\xi)\right\|^{2} \leq B$. By Theorem 1.8 and (2.1), the proof is completed.

In Theorem 2.5, using the Gramian operators we establish necessary and sufficient conditions that $X=\left\{\tau_{[s]} \phi ; \phi \in \Phi, \quad[s] \in G / H\right\}$ to be a frame. For this we need the following lemma. We denote $\Phi \times G / H=\mathcal{I}$, where $\Phi$ is an at most countable subset of $L^{2}(G)$. 
Lemma 2.3. Let $R: H^{\perp} \rightarrow B\left(l^{2}(\Phi)\right)$ be a weakly measurable operator such that $\int_{H^{\perp}}\left|\left\langle R(\xi) \delta_{\phi_{1}}, \delta_{\phi_{2}}\right\rangle\right| d \nu(\xi)<\infty$, where $\Phi$ is an at most countable subset of $L^{2}(G)$ and $\left\{\delta_{\phi}\right\}_{\phi \in \Phi}$ is the standard orthonormal basis for $l^{2}(\Phi)$. Then the following are equivalent:

(i) The integral $\int_{H^{\perp}} \sum_{\phi_{1}, \phi_{2} \in \Phi^{\prime}}\left\langle R(\xi) \delta_{\phi_{1}}, \delta_{\phi_{2}}\right\rangle m_{\phi_{1}}(\xi) \overline{m_{\phi_{2}}(\xi)} d \nu(\xi) \geq 0$ holds for any finite set $\Phi^{\prime} \subset \Phi$ and any trigonometric polynomial

$$
m_{\phi}(\xi)=\sum_{[s] \in G / H} c_{[s], \phi} \overline{\omega_{[s]}(\xi)}
$$

with finite nonzero coefficients $c_{[s], \phi}$.

(ii) $R(\xi) \geq 0$ for a.e. $\xi \in H^{\perp}$.

Proof. If $x(\xi)=\sum_{\phi \in \Phi^{\prime}} m_{\phi}(\xi) \delta_{\phi}$, then

$$
\sum_{\phi_{1}, \phi_{2} \in \Phi^{\prime}}\left\langle R(\xi) \delta_{\phi_{1}}, \delta_{\phi_{2}}\right\rangle m_{\phi_{1}}(\xi) \overline{m_{\phi_{2}}(\xi)}=\langle R(\xi) x(\xi), x(\xi)\rangle .
$$

By (2.2), (ii) implies (i).

Conversely, let (i) holds. If (ii) fails, then there exists a measurable subset $E$ of $H^{\perp}$ such that $\nu(E)>0$ and $\langle R(\xi) Y, Y\rangle<0$, where $Y \in l^{2}(\Phi)$, with only finitely many components. Also we assume that $\left\{y_{\phi}\right\}_{\phi \in \Phi}$ is dense in $l^{2}(\Phi)$ (note that $l^{2}(\Phi)$ is separable) such that for each $\phi,\left\langle y_{\phi}, \delta_{\phi_{i}}\right\rangle=0$ except for finitely many $\phi \in \Phi$. For proving $R(\xi) \geq 0$, we must show that $\left\langle R(\xi) y_{\phi}, y_{\phi}\right\rangle \geq 0$ for a.e. $\xi \in H^{\perp}$. By Luzin's Theorem [18], there exists a uniformly bounded sequence of trigonometric polynomials $\left\{h_{j}\right\}_{j \geq 1}$ that is pointwise convergence to the characteristic function $\chi_{E}$ on $H^{\perp}$. Then we have

$$
\int_{H^{\perp}}\langle R(\xi) u(\xi), u(\xi)\rangle d \nu(\xi)=\int_{E}\langle R(\xi) Y, Y\rangle d \nu(\xi)<0,
$$

where $u(\xi)=\chi(\xi) Y \in l^{2}(\Phi)$. Set $u_{j}=h_{j}(\xi) Y \in l^{2}(\Phi)$. Thus

$$
\int_{H^{\perp}}\left\langle R(\xi) u_{j}(\xi), u_{j}(\xi)\right\rangle d \nu(\xi)=\int_{H^{\perp}}\left|h_{j}\right|^{2}\langle R(\xi) Y, Y\rangle d \nu(\xi),
$$

by (2.2), Lebesgue dominated convergence Theorem implies that (2.4) converges to left-hand side of (2.3). Therefore if $j \rightarrow \infty$, then (2.4) is negative.

Define finite subset $\Phi_{1}$ of $\Phi$ by $\left\{\phi \in \Phi ; y_{\phi} \neq 0\right\}$ and $m_{\phi}^{j}=y_{\phi} h_{j}(\xi)$, then by (2.4) we have

$$
\int_{H^{\perp}} \sum_{\phi_{1}, \phi_{2} \in \Phi_{1}}\left\langle R(\xi) \delta_{\phi_{1}}, \delta_{\phi_{2}}\right\rangle m_{\phi_{1}}^{j}(\xi) \overline{m_{\phi_{2}}^{j}(\xi)} d \nu(\xi)<0 .
$$

This is a contradiction with (i), and the proof is completed.

Theorem 2.4. We assume that $X=\left\{\tau_{[s]} \phi ; \phi \in \Phi, \quad[s] \in G / H\right\}$. Then the following are equivalent:

(1) The system $X$ is a frame with constants $A, B$ for its closed linear span $V_{\Phi}$. 
(2) $A \mathbb{G}(\xi) \leq \mathbb{G}^{2}(\xi) \leq B \mathbb{G}(\xi)$ for a.e. $\xi \in H^{\perp}$.

(3) $A \widetilde{\mathbb{G}}(\xi) \leq \widetilde{\mathbb{G}}^{2}(\xi) \leq B \widetilde{\mathbb{G}}(\xi)$ for a.e. $\xi \in H^{\perp}$.

Proof. We must show that $\left\{\tau_{[s]} \phi ; \quad[s] \in G / H\right\}$ is a frame for dense subset $M_{0}$ of $V_{\Phi}$ (see Lemma 5.1 .7 of [6]). If $f \in M_{0}$, then $f=\sum_{([s], \phi) \in \mathcal{I}} c_{[s], \phi} \tau_{[s]} \phi$, where only finitely many of $c_{[s], \phi} \neq 0$. Thus $\hat{f}(\xi)=\sum_{([s], \phi) \in \mathcal{I}} c_{[s], \phi} \omega_{[s]}(\xi) \hat{\phi}(\xi)=$ $\sum_{\phi \in \Phi} m_{\phi}(\xi) \hat{\phi}(\xi)$, where $m_{\phi}$ is defined in Lemma 2.3. Let (1) holds by Theorem 2.2, esssup $\|G(\xi)\| \leq B$. Thus $G(\xi) \in B\left(l^{2}(\Phi)\right)$. With a simple computation (for more details see [2]) we have

$$
\sum_{\phi \in \Phi} \sum_{[s] \in G / H}\left|\left\langle\tau_{[s]} \phi, f\right\rangle\right|^{2}=\sum_{\phi_{1} \in \Phi} \int_{H^{\perp}}\left|\left\langle\digamma \phi_{1}(\xi), \digamma f(\xi)\right\rangle_{l_{\perp}^{2}}\right|^{2} d \nu(\xi) .
$$

Then,

$$
\begin{aligned}
& \sum_{\phi_{1} \in \Phi} \int_{H^{\perp}}\left|\left\langle\digamma \phi_{1}(\xi), \digamma f(\xi)\right\rangle_{l_{\perp}^{2}}\right|^{2} d \nu(\xi) \\
= & \sum_{\phi_{1} \in \Phi} \int_{H^{\perp}}\left|\sum_{\phi_{2} \in \Phi} m_{\phi_{2}}(\xi)\left\langle\digamma\left(\phi_{2}\right)(\xi), \digamma \phi_{1}(\xi)\right\rangle\right|^{2} d \nu(\xi) \\
= & \int_{H^{\perp}} \sum_{\phi_{1}, \phi_{2}, \phi_{3} \in \Phi} \mathbb{G}_{\phi_{1}, \phi_{2}}^{*}(\eta) \mathbb{G}_{\phi_{2}, \phi_{3}}(\eta) m_{\phi_{1}}(\eta) \overline{m_{\phi_{2}}}(\eta) d \nu(\eta) .
\end{aligned}
$$

Since $\mathbb{G}$ is self-adjoint then the right-hand of $(2.5)$ is equal to

$$
\int_{H^{\perp}} \sum_{\phi_{1}, \phi_{2} \in \Phi}\left\langle\mathbb{G}^{2}(\eta) \delta_{\phi_{1}}, \delta_{\phi 2}\right\rangle m_{\phi_{1}}(\eta) \overline{m_{\phi_{2}}(\eta)} d \nu(\eta) .
$$

Now, Weil's Formula and Plancherel Theorem imply that

$$
\begin{aligned}
\|f\|^{2} & =\|\hat{f}\|^{2} \\
& =\int_{\hat{G}}\left|\sum_{\phi \in \Phi} m_{\phi}(\xi) \hat{\phi}(\xi)\right|^{2} d \nu(\xi) \\
& =\int_{H^{\perp}} \int_{\hat{G} / H^{\perp}}\left|\sum_{\phi \in \Phi} m_{\phi}(\xi) \hat{\phi}(\xi)\right|^{2} d \nu(\xi) \\
& =\int_{H^{\perp}} \sum_{\phi_{1}, \phi_{2} \in \Phi} \sum_{[\eta] \in \hat{G} / H^{\perp}} \hat{\phi}_{1}(\eta+\xi) \overline{\hat{\phi}_{2}(\eta+\xi)} m_{\phi_{1}}(\xi) \overline{m_{\phi_{2}}(\xi)} d \nu(\xi) \\
& =\int_{H^{\perp}} \sum_{\phi_{1}, \phi_{2} \in \Phi} \mathbb{G}(\xi) m_{\phi_{1}}(\xi) \overline{m_{\phi_{2}}(\xi)} d \nu(\xi) .
\end{aligned}
$$


Using pervious lemma, (2.6) and (2.7) we have (2) is equivalent to (1). First we assume that $R(\xi)=B \mathbb{G}(\xi)-\mathbb{G}^{2}(\xi)$ and second $R(\xi)=\mathbb{G}^{2}(\xi)-A \mathbb{G}(\xi)$. Since $\mathbb{G}(\xi)=K^{*}(\xi) K(\xi)$ and $\widetilde{G}(\xi)=K(\xi) K^{*}(\xi)$, then $K^{*}(\xi) K(\xi) K^{*}(\xi) K(\xi) \geq$ $A K^{*}(\xi) K(\xi)$, which implies that $\widetilde{\mathbb{G}}^{3}(\xi) \geq A \widetilde{\mathbb{G}}^{2}(\xi)$. Therefore, it shows that $(2)$ and (3) are equivalent.

The following theorems yield directly from definition of the pre-Gramian operator and the Gramian operator. The next theorem present a necessary and sufficient condition that the shifts of a countable subset of $L^{2}(G)$ is a frame for $L^{2}(G)$.

Theorem 2.5. Let $X=\left\{\tau_{[s]} \phi ; \quad[s] \in G / H, \phi \in \Phi\right\}$ be a frame sequence with bounds $A$ and $B$. Then the following are equivalent:

(1) $X$ is a frame for $L^{2}(G)$.

(2) $\widetilde{\mathbb{G}}(\xi)\left(K^{*}(\xi)\right)$ is injective for a.e. $\xi \in H^{\perp}$.

(3) $A I \leq \widetilde{\mathbb{G}}(\xi) \leq$ BIfor a.e. $\xi \in H^{\perp}$.

Proof. By definitions of the dual Gramian operator, $\widetilde{\mathbb{G}}(\xi)=K^{*}(\xi) K(\xi)$ and the pre-Gramian operator $K$ we have

$$
\langle\widetilde{\mathbb{G}}(\xi) f, f\rangle=\langle K(\xi) f, K(\xi) f\rangle=\sum_{(\phi,[s]) \in \mathcal{I}}\left|\left\langle\tau_{[s] \phi}, f\right\rangle\right|^{2} .
$$

So (1), (2) and (3) are equivalent.

Example 2.1. For a prime number $p$. Let $G=\mathbb{Q}_{p}$ be the $p$-adic group with compact open subgroup $H=\mathbb{Z}_{p}$. The group $G$ is self dual $[9]$ thus $\hat{G}=\mathbb{Q}_{p}$ and $H^{\perp}=\mathbb{Z}_{p}$. For $\sigma \in \mathcal{D}, \hat{\phi}_{\sigma}=1_{\sigma+\mathbb{Z}_{p}}$ and then $\widetilde{\mathbb{G}}(\xi)=I$. By Theorem 2.5, $X=\left\{\tau_{[s]} \phi_{\sigma} ; \quad[s] \in \mathbb{Q}_{p} / \mathbb{Z}_{p}, \quad \sigma \in \mathcal{D}\right\}$ forms a frame for $L^{2}\left(\mathbb{Q}_{p}\right)$.

In the following theorem we state a necessary and sufficient condition for $X=\left\{\tau_{[s]} \phi ; \quad[s] \in G / H, \phi \in \Phi\right\}$ to be a Riesz basis.

Theorem 2.6. Let $X=\left\{\tau_{[s]} \phi ; \quad[s] \in G / H, \phi \in \Phi\right\}$ be a frame sequence with bounds $A$ and $B$. Then the following are equivalent:

(1) $X$ is a Riesz basis for $V_{\Phi}$.

(2) $\mathbb{G}(\xi)(K(\xi))$ is injective for a.e. $\xi \in H^{\perp}$.

(3) $A I \leq \mathbb{G}(\xi) \leq B I$ for a.e. $\xi \in H^{\perp}$.

Proof. By definitions of $\mathbb{G}$ and $K^{*}$ we have

$$
\langle\mathbb{G}(\xi) c, c\rangle=\left\langle K^{*}(\xi) c, K^{*}(\xi) c\right\rangle=\left\|\sum_{\phi,[s] \in \mathcal{I}} c_{\phi,[s]} \digamma \tau_{[s]} \phi(\xi)\right\|^{2} .
$$

Thus (1), (2) and (3) are equivalent. 
Example 2.2. We assume that $G=\mathbb{Q}_{3}$ and $H=\mathbb{Z}_{3}$. Also $\hat{G}=\mathbb{Q}_{3}$ and $H^{\perp}=$ $\mathbb{Z}_{3}$ (see Example 2.1). If $\hat{\phi}_{1}=1_{\mathbb{Z}_{3} \cup\left(1+\mathbb{Z}_{3}\right)}$ and $\hat{\phi}_{2}=1_{2+\mathbb{Z}_{3}}$, then $\mathbb{G}(\xi)=\left(\begin{array}{ll}2 & 0 \\ 0 & 1\end{array}\right)$. By Theorem 2.6, $X=\left\{\tau_{[s]} \phi_{1}, \tau_{[s]} \phi_{2} ; \quad[s] \in \mathbb{Q}_{3} / \mathbb{Z}_{3}\right\}$ forms a Riesz basis for $V_{\Phi}=\overline{\operatorname{span}} X$.

In the following example we present a collection which is a frame sequence but it is NOT a Riesz basis for closure of its span.

Example 2.3. Consider the 3 -adic group $\mathbb{Q}_{3}$ with compact open subgroup $\mathbb{Z}_{3}$. If $V_{1}=3 \mathbb{Z}_{3}, V_{2}=1+3 \mathbb{Z}_{3}$ and $V_{3}=2+3 \mathbb{Z}_{3}$, then $\left\{V_{i} ; i=1,2,3\right\}$ is a partition for $\mathbb{Z}_{3}[3]$. Let $\hat{\phi}_{1}=1_{V_{1}}$ and $\hat{\phi}_{2}=1_{V_{3}}$. Thus

$$
\begin{gathered}
\mathbb{G}(\xi)=\left(\begin{array}{ll}
1 & 0 \\
0 & 0
\end{array}\right) \text { for } \xi \in 3 \mathbb{Z}_{3}, \quad \mathbb{G}(\xi)=\left(\begin{array}{ll}
0 & 0 \\
0 & 0
\end{array}\right) \text { for } \xi \in 1+3 \mathbb{Z}_{3}, \\
\mathbb{G}(\xi)=\left(\begin{array}{ll}
0 & 0 \\
0 & 1
\end{array}\right) \text { for } \xi \in 2+3 \mathbb{Z}_{3} .
\end{gathered}
$$

Since $\nu\left(1+3 \mathbb{Z}_{3}\right)=1$, then the Gramian operator $\mathbb{G}(\xi)$ is not injective. By Theorem 2.6, $\left\{\tau_{[s]} \phi_{1}, \tau_{[s]} \phi_{2} ;[s] \in \mathbb{Q}_{3} / \mathbb{Z}_{3}\right\}$ is not Riesz basis for $V_{\Phi}$.

\section{Shift generated dual frames in $L^{2}(G)$}

Our main goal in this section is to generalize a characterization of SG-dual frame for shift invariant subspaces of $L^{2}(G)$ by the Gramian operators and range functions. First, we need to establish the following lemma. The function $h \in L^{2}(G)$ is called $\mathcal{D}$-periodic function whenever $f(\xi+\eta)=f(\xi), \eta \in \mathcal{D}$ and for a.e. $\xi \in H^{\perp}$. For example, the function $\omega_{[s]}$ is a $\mathcal{D}$-periodic function.

Lemma 3.1. Let $m_{\phi}$ be a measurable $\mathcal{D}$-periodic function for $\phi \in \Phi$ such that $\int_{H^{\perp}} \sum_{\phi \in \Phi}\left|m_{\phi}(\xi)\right|^{2} d \nu(\xi)<\infty$. Then for $X=\left\{\tau_{[s]} \phi ; \quad \phi \in \Phi,[s] \in G / H\right\}$ with the Bessel property, the following are equivalent:

(a) $\sum_{\phi \in \Phi} \int_{H^{\perp}}\langle\digamma f(\xi), \digamma \phi(\xi)\rangle \overline{m_{\phi}(\xi)} d \nu(\xi)=0$ for all $f \in V_{\Phi}$;

(b) For a.e. $\xi \in H^{\perp}$, we have $\sum_{\phi \in \Phi}\langle\digamma f(\xi), \digamma \phi(\xi)\rangle \overline{m_{\phi}(\xi)} d \nu(\xi)=0$ for all $f \in V_{\Phi}$.

Proof. Let (a) holds. If $\left\{e_{[\eta]}\right\}_{[\eta] \in \hat{G} / H^{\perp}}$ is the standard orthonormal basis for $l_{\perp}^{2}$, then $\digamma f(\xi)=\sum_{[\eta] \in \hat{G} / H^{\perp}} P(\xi) e_{[\eta]}$, where $P(\xi): l_{\perp}^{2} \rightarrow J(\xi)$ is an orthogonal projection and $J(\xi)$ is a range function. Thus

$$
\sum_{\phi \in \Phi}\left\langle\sum_{[\eta] \in \hat{G} / H^{\perp}} P(\xi) e_{[\eta]}, \digamma \phi(\xi)\right\rangle=\sum_{\phi \in \Phi}\langle\digamma f(\xi), \digamma \phi(\xi)\rangle=0 .
$$

Now we assume that (b) fails. Then there exists a measurable subset $E$ of $H^{\perp}$ such that $h(\xi)=\sum_{[\eta] \in \hat{G} / H^{\perp}}\left\langle P(\xi) e_{[\eta]}, \digamma \phi(\xi)\right\rangle \overline{m_{\phi}(\xi)} \neq 0$. Therefore, we have the subsets

$$
\begin{aligned}
& E_{1}=\{\xi \in E ; \quad \operatorname{Re} h(\xi)>0\}, \quad E_{2}=\{\xi \in E ; \quad \operatorname{Im} h(\xi)>0\}, \\
& E_{3}=\{\xi \in E ; \operatorname{Re} h(\xi)<0\}, \quad E_{4}=\{\xi \in E ; \quad \operatorname{Im} h(\xi)<0\} .
\end{aligned}
$$


Set $\digamma f_{1}=\chi_{E_{1}} e_{[\eta]}$, then $f_{1} \in V_{\Phi}$ and $\sum_{\phi \in \Phi} \int_{H^{\perp}}\left\langle\digamma f_{1}(\xi), \digamma \phi(\xi)\right\rangle \overline{m_{\phi(\xi)}} d \nu(\xi) \neq$ 0 . That is a contradiction and proof is completed. It is clear that (b) implies (a).

In the following theorems and corollary we characterize the shift generated dual frame for shift invariant space which forms a frame.

Theorem 3.2. We assume that $X=\left\{\tau_{[s]} \phi ; \quad[s] \in G / H, \phi \in \Phi\right\}$ is a frame for closed subspace $V_{\Phi} \subseteq L^{2}(G)$ and $R: \Phi \rightarrow L^{2}(G)$ is a mapping that the collection $Y=\left\{\tau_{[s]} R \phi ; \quad[s] \in G / H, \phi \in \Phi\right\}$ is Bessel, then $Y$ is a SG-dual frame for $X$ if and only if the collection $\tilde{Y}=\{\digamma R \phi(\xi)\}_{\phi \in \Phi}$ is a SG-dual frame for $\widetilde{X}=\{\digamma \phi(\xi)\}_{\phi \in \Phi}$.

Proof. By Plancherel Theorem and Weil's Formula we have

$$
\|f\|^{2}=\|\hat{f}\|^{2}=\int_{\hat{G}}|\hat{f}(\xi)|^{2} d \nu(\xi)=\int_{H^{\perp}} \sum_{[\eta] \in \hat{G} / H^{\perp}}|\hat{f}(\xi+\eta)|^{2} d \nu(\xi) .
$$

Let $\widetilde{Y}$ be a SG-dual frame for $\widetilde{X}$, thus for a.e. $\xi \in H^{\perp}$,

$$
\sum_{\phi \in \Phi}\langle\digamma f(\xi), \digamma \phi(\xi)\rangle \overline{\langle\digamma g(\xi), \digamma R \phi(\xi)\rangle}=\langle\digamma f(\xi), \digamma g(\xi)\rangle .
$$

We must show that $\sum_{(\phi,[s]) \in \mathcal{I}}\left\langle f, \tau_{[s]} \phi\right\rangle \overline{\left\langle g, \tau_{[s]} R \phi\right\rangle}=\langle f, g\rangle$.

For $f \in V_{\Phi}$,

$$
\begin{aligned}
& \sum_{(\phi,[s]) \in \mathcal{I}}\left\langle f, \tau_{[s]} \phi\right\rangle \overline{\left\langle g, \tau_{[s]} R \phi\right\rangle} \\
& =\sum_{(\phi,[s]) \in \mathcal{I}}\left(\int_{\hat{G}} \hat{f}(\xi) \omega_{[s]}(\xi) \widehat{\widehat{\phi(\xi)}} d \nu(\xi) \times \int_{\hat{G}} \overline{\hat{g}(\xi)} \overline{\omega_{[s]}}(\xi) \widehat{R \phi(\xi)} d \nu(\xi)\right) \\
& =\sum_{(\phi,[s]) \in \mathcal{I}}\left(\int_{H^{\perp}} \sum_{[\eta] \in \hat{G} / H^{\perp}} \hat{f}(\xi+\eta) \omega_{[s]}(\xi) \overline{\phi(\hat{\xi+\eta})} d \nu(\xi)\right. \\
& \left.\left.\times \int_{H^{\perp}} \sum_{[\eta] \in \hat{G} / H^{\perp}} \overline{\hat{g}(\xi+\eta)} \overline{\omega_{[s]}}(\xi) R \widehat{\phi(\xi+\eta}\right) d \nu(\xi)\right) \\
& =\sum_{\phi \in \Phi}\left(\int_{H^{\perp}}\langle\digamma f(\xi), \digamma \phi(\xi)\rangle \omega_{[s]}(\xi) d \nu(\xi) \times \overline{\int_{H^{\perp}}\langle\digamma g(\xi), \digamma R \phi(\xi)\rangle \overline{\omega_{[s]}(\xi)} d \nu(\xi)}\right) \\
& =\sum_{\phi \in \Phi}\langle\digamma f \widehat{(\xi), \digamma} \phi(\xi)\rangle \overline{\langle\digamma g(\xi), \digamma R \phi(\xi)\rangle},
\end{aligned}
$$

the Parseval's identity [18] implies that

$$
\sum_{\phi \in \Phi}\langle\digamma f \widehat{(\xi), \digamma} \phi(\xi)\rangle \widehat{\langle\digamma g(\xi), \digamma R \phi(\xi)\rangle}=\langle\digamma f(\xi), \digamma g(\xi)\rangle_{L_{*}^{2}}
$$




$$
=\int_{H^{\perp}}\langle\digamma f(\xi), \digamma g(\xi)\rangle_{l_{\perp}^{2}} d \nu(\xi) .
$$

By Weil's Formula and Plancherel Theorem we have

$$
\begin{aligned}
\int_{H^{\perp}}\langle\digamma f(\xi), \digamma g(\xi)\rangle d \nu(\xi) & =\int_{H^{\perp}} \sum_{[\eta] \in \hat{G} / H^{\perp}} f(\hat{\xi+\eta}) \overline{g(\hat{\xi+\eta})} d \nu(\xi) \\
& =\int_{\hat{G}} f \hat{f} \bar{\xi} \overline{g \hat{(\xi)}} d \nu(\xi)=\langle\hat{f}, \hat{g}\rangle_{L^{2}(\hat{G})}=\langle f, g\rangle_{L^{2}(G)} .
\end{aligned}
$$

Therefore, $Y$ is a SG-dual frame for $X$. For the converse, let $Y$ be SG-dual frame for $X$, then

$$
\sum_{(\phi,[s]) \in \mathcal{I}}\left\langle f, \tau_{[s]} \phi\right\rangle \overline{\left\langle g, \tau_{[s]} R \phi\right\rangle}=\langle f, g\rangle .
$$

We must show that for all $f, g \in V_{\Phi}$

$$
\sum_{\phi \in \Phi}\langle\digamma f(\xi), \digamma \phi(\xi)\rangle \overline{\langle\digamma g(\xi), \digamma R \phi(\xi)\rangle}=\langle\digamma f(\xi, \digamma g(\xi))\rangle \text { for a.e. } \xi \in H^{\perp} \text {. }
$$

Let (3.1) fails. Then there exists a measurable subset $E_{1}$ of $H^{\perp}$ with $\nu\left(E_{1}\right)>0$ and $\left[\eta_{1}\right],\left[\eta_{2}\right] \in \hat{G} / H^{\perp}$, such that for a.e. $\xi \in E_{1}$,

$$
\mathcal{F}(\xi)=\left(\sum_{\phi \in \Phi}\left\langle P(\xi) e_{\left[\eta_{1}\right]}, \digamma \phi(\xi)\right\rangle \overline{\left\langle P(\xi) e_{\left[\eta_{2}\right]}, \digamma R \phi(\xi)\right\rangle}-\left\langle P(\xi) e_{\left[\eta_{1}\right]}, P(\xi) e_{\left[\eta_{2}\right]}\right\rangle\right) \neq 0 .
$$

Thus one of the following inequality holds

$$
\operatorname{Re}(\mathcal{F}(\xi))>0, \quad \operatorname{Re}(\mathcal{F}(\xi))<0, \quad \operatorname{Im}(\mathcal{F}(\xi))>0, \quad \operatorname{Im}(\mathcal{F}(\xi))<0 \text { for } \quad \xi \in E_{1} .
$$

It is easy to show that all of these cases do not hold [12]. Therefore proof is completed.

By using Theorem 3.2 and Theorem 2.5, we have the following corollary.

Corollary 3.3. Retain the assumption of pervious theorem. Then, $Y$ is a $S G$ dual frame of type I for $X$ if and only if the collection $\widetilde{Y}=\{\digamma R \phi(\xi)\}_{\phi \in \Phi}$ is a $S G$-dual frame of type I frame for $\widetilde{X}=\{\digamma \phi(\xi)\}_{\phi \in \Phi}$.

We use the idea of Lemma 2 of [12] and Lemma 3.1, to prove following theorem.

Theorem 3.4. Consider the assumption of Theorem 3.2. Then, $Y$ is a $S G$ dual of type II for $X$ if and only if the collection $\widetilde{Y}=\{\digamma R \phi(\xi)\}_{\phi \in \Phi}$ is a $S G$-dual frame of type II for $\widetilde{X}=\{\digamma \phi(\xi)\}_{\phi \in \Phi}$.

Theorem 3.5 provides us necessary and sufficient conditions for standard dual $X=\left\{\tau_{[s]} \phi ; \quad[s] \in G / H, \phi \in \Phi\right\}$ to be a unique SG-dual frame of type I or type II, in terms of the Gramian operator and range functions. For proof of the following theorem we refer to Theorems 6 and 7 of [12]. 
Theorem 3.5. We assume that $X=\left\{\tau_{[s]} \phi ; \quad[s] \in G / H, \phi \in \Phi\right\}$ is a frame with frame bounds $A$ and $B$ for the closed subspace $V_{\Phi} \subseteq L^{2}(G)$. Then the following are equivalent.

(1) The standard dual is the unique SG-dual frame of type I (resp. type II) for $X$.

(2) We have either $J(\xi)=0$ or $\{\digamma \phi(\xi)\}_{\phi \in \Phi}$ is a Riesz basis for $J(\xi)$ (a frame for $\left.L^{2}(G)\right)$ for a.e. $\xi \in H^{\perp}$.

(3) We have either $\mathbb{G}(\xi)=0$ (resp. $\widetilde{\mathbb{G}}(\xi)=0$ ) or $A I \leq \mathbb{G}(\xi) \leq B I$ (resp. $A I \leq \widetilde{\mathbb{G}}(\xi) \leq B I)$ for a.e. $\xi \in H^{\perp}$.

\section{References}

[1] A. Ahmadi, A. A. Hemmat, and R. R. Tousi, Shift invariant spaces for local fields, Int. J. Wavelets Multiresolut. Inf. Process. 9 (2011), no. 3, 417-426.

$[2] \_$A A characterization of shift invariant spaces on LCA group $G$ with a compact open subgroup, preprint.

[3] J. J. Benedetto and R. L. Benedetto, A wavelet theory for local fields and related groups, J. Geom. Anal. 14 (2004), no. 3, 423-456.

[4] R. L. Benedetto, Examples of wavelets for local fields, Wavelets, frames and operator theory, 27-47, Contemp. Math., 345, Amer. Math. Soc., Providence, RI, 2004.

[5] M. Bownik, The structure of shift invariant subspaces of $L^{2}\left(\mathbb{R}^{n}\right)$, J. Funct. Anal. 177 (2000), no. 2 282-309.

[6] O. Christensen, An Introduction to Frames and Riesz Bases, Birkhäuser, Boston, 2003.

[7] I. Daubechies, A. Grossmann, and Y. Meyer, Painless nonorthogonal expansion, J. Math. Phys. 27 (1986), no. 5, 1271-1283.

[8] R. J. Duffin and A. C. Schaefer, A class of nonharmonic Fourier series, Trans. Amer. Math. Soc. 72 (1952), 341-366.

[9] G. B. Folland, A Course in Abstract Harmonic Analysis, CRC Press, 1995.

[10] P. H. Frampton and Y. Okada, P-adic string N-point function, Phys. Rev. Lett. B 60 (1988), 484-486.

[11] H. Helson, Lectures on Invariant Subspaces, Academic Press, New York, London, 1964.

[12] A. A. Hemmat and J. P. Gabardo, The uniqueness of shift-generated duals for frames in shift-invariant subspaces, J. Fourier Anal. Appl. 13 (2007), no. 5, 589-606.

[13] E. Hewitt and K. A. Ross, Abstract Harmonic Analysis. Vol. I: Structure of Topological Groups. Integration Theory, Group Representations, Springer, Berlin, 1963.

[14] R. A. Kamyabi Gol and R. R. Tousi, The structure of shift invariant spaces on locally compact abelian group, J. Math. Anal. Appl. 340 (2008), 219-225.

[15] _ A range function approach to shift invariant spaces on locally compact abelian group, Int. J. Wavelets Multiresolut. Inf. Process 8 (2010), no. 1, 49-59.

[16] N. J. Munch, Noise reduction in tight Weyl-Heisenberg frames, IEEE Trans. Inform. Theory 38 (1992), no. 2, part 2, 608-616.

[17] H. Reiter and J. D. Stegeman, Classical Harmonic Analysis and Locally Compact Groups, Clarendon Press. Oxford, 2000.

[18] W. Rudin, Real and Complex Analysis, McGraw-Hill Co., Singapore, 1987.

Ahmad Ahmadi

Department of Mathematics

HORMOZGAN UNIVERSITY

BANDAR ABBAS 3995, IRAN

E-mail address: ahmadi@mail.vru.ac.ir 
Ataollah Askari-Hemmat

Department of Mathematics

Kerman Graduate University of Technology

KERMAN 7631133131, IRAN

AND

Department of Mathematics

ShaHid BaHonaR UNIVERSity OF KERMAN

KERMAN 7616914111, IRAN

E-mail address: askari@mail.uk.ac.ir 